\title{
BOUNDED AND ALMOST PERIODIC SOLUTIONS AND EVOLUTION SEMIGROUPS ASSOCIATED WITH NONAUTONOMOUS FUNCTIONAL DIFFERENTIAL EQUATIONS
}

\author{
BERND AULBACH AND NGUYEN VAN MINH
}

Received 8 September 2000

We study evolution semigroups associated with nonautonomous functional differential equations. In fact, we convert a given functional differential equation to an abstract autonomous evolution equation and then derive a representation theorem for the solutions of the underlying functional differential equation. The representation theorem is then used to study the boundedness and almost periodicity of solutions of a class of nonautonomous functional differential equations.

\section{Introduction}

This paper is concerned with evolution semigroups associated with nonautonomous functional differential equations and their applications to study the asymptotic behavior of solutions to the underlying equations. Recently, this method has been extensively employed to study the asymptotic behavior of evolutionary processes determined by differential equations. Among the references listed in this paper we refer the reader to $[1,13,15,17,19]$ and the references therein for applications of this method to study the wellposedness, stability, and exponential dichotomy of evolutionary processes determined by evolution equations without delay. In [18], we have demonstrated another useful application of evolution semigroup method to find spectral criteria for almost periodicity of solutions of linear periodic evolution equations.

In contrast to the above-mentioned works which are mainly concerned with linear or semilinear evolution equations without delay, in this paper, we deal with nonlinear nonautonomous functional differential equations. We describe thoroughly the evolution semigroups associated with underlying equations and prove a representation theorem for solutions of equations under consideration. We then demonstrate an application of the obtained results to study the boundedness and almost periodicity of solutions to functional differential equations. As a result we get a sufficient condition for the existence of bounded and almost periodic solutions which is an extension to fully nonlinear functional differential equations of previous results by other authors (see [10, 12, 16, 20, 21] 
for related results and methods, $[6,25]$ for more information on ordinary differential equations with almost periodic coefficients, and [8] for various conditions for almost periodicity of solutions of equations with infinite delay). In this paper, we put an emphasis on an application of evolution semigroup method to the study of boundedness and almost periodicity of solutions to nonlinear nonautonomous functional differential equations via nonlinear semigroup techniques. For other various applications of nonlinear semigroup theory to nonlinear functional differential equations, we refer the reader to $[5,8,9,22,24]$ and the references therein for some fundamental notions and results which may have direct relations with the present paper. We now give a brief outline of our constructions. Under the assumptions of the global existence and uniqueness theorem, the Functional Differential Equations (FDE) under consideration generates an evolutionary process $\{U(t, s), t \geq s\}$. To this process we associate the so-called evolution semigroup of (possibly nonlinear) operators $\left\{T^{h}, h \geq 0\right\}$ defined by the formula

$$
\left(T^{h} v\right)(t)=U(t, t-h) v(t-h),
$$

where $v$ belongs to a suitable space of functions. The key point of our study is the analysis of the semigroup $\left\{T^{h}, h \geq 0\right\}$ and its generator in the framework of the Crandall-Liggett theorem [3, Theorem 1] and the Brezis-Pazy theorem [2, Corollary 4.3]. It turns out that the infinitesimal generator of this semigroup can be computed explicitly and that it generates the semigroup in the sense of Crandall and Liggett [3]. As an application of this, we then consider the asymptotic behavior of solutions of the FDE under consideration. We exploit the resemblance of the operator $T^{h}$ with the monodromy operator of a differential equation with periodic coefficients and derive a sufficient condition for the existence of exponentially stable bounded and almost periodic solutions. Our condition is given in terms of the accretiveness of the operator $A$ defined below. In the case of equations without delay the operator $A$ turns out to be the differential operator $-d / d t+f(t, \cdot)$. Thus, in this case, the accretiveness of $A$ follows from that of $f(t, \cdot)$ for all $t$. In light of this, our result seems to be new for nonautonomous functional differential equations (see [10, 12, 16, 21, 20] for closely related results and methods). Moreover, our condition is given in other form than that of the above-mentioned papers, which has a direct relation to those of Bohl-Perron type for exponential dichotomy of linear equations (see [4, Chapters III and IV] for more information).

\section{Preliminaries}

In this section, we collect some well-known notions as well as some results which will be used throughout this paper.

Definition 2.1. Let $Y$ be an arbitrary Banach space and $A$ a (single-valued) operator acting in $Y$. Then $A$ is said to be accretive if the estimate

$$
\|(I+\lambda A) x-(I+\lambda A) y\| \geq\|x-y\|
$$

is true for all $x, y \in D(A)$ and $\lambda>0$. 
The following result about accretive operators will be useful later on. Its proof can be found in [3].

Lemma 2.2. Let $A$ be an operator acting in a Banach space $Y$. Then the operator $(\omega I-A)$ is accretive if and only if

$$
\|(I-\lambda A) x-(I-\lambda A) y\| \geq(1-\lambda \omega)\|x-y\|
$$

for all $x, y \in D(A)$ and $\lambda>0$.

Definition 2.3. A family $\{T(t), t \geq 0\}$ of mappings on a subset $C \subset Y$ is said to be a strongly continuous semigroup of type $\omega$ if the following conditions hold:

(i) $T(0) x=x$ for all $x \in C$,

(ii) $T(t) x$ is continuous in $t$ for each fixed $x \in C$,

(iii) $T\left(t_{1}+t_{2}\right)=T\left(t_{1}\right)+T\left(t_{2}\right)$ for all $t_{1}, t_{2} \geq 0$,

(iv) $\|T(t) x-T(t) y\| \leq e^{\omega t}\|x-y\|$ for all $x, y \in C, t \geq 0$.

The results being derived in this paper rely upon the following general theorems which are due to M. Crandall and T. Liggett [3] and H. Brezis and A. Pazy [2], respectively. For the reader's convenience we state them in full details.

Theorem 2.4 (M. Crandall and T. Liggett [3, Theorem 1]). Let $\omega$ be a real number and let $B$ be a densely defined operator acting in $Y$ such that $\omega I+B$ is accretive. In addition, suppose that there exists a positive number $\lambda_{0}$ such that $R(I+\lambda B)=Y$ for all $0<\lambda<\lambda_{0}$. Then $-B$ generates a strongly continuous semigroup $\{S(t), t \geq 0\}$ on $Y$ which for all $x \in Y, n \in \mathbb{N}$ and $t \geq 0$ satisfies the two conditions:

(i) $(I+(t / n) B)^{-n} x=S(t) x$,

(ii) $\|S(t) x-S(t) y\| \leq e^{\omega t}\|x-y\|$.

Theorem 2.5 (Adaption from H. Brezis and A. Pazy [2, Corollary 4.3]). Let $\{T(t), t \geq$ $0\}$ be a family of mappings from a Banach space $Y$ into itself such that

$$
\|T(t) x-T(t) y\| \leq M(t)\|x-y\| \quad \forall t \geq 0, x, y \in Y,
$$

where $M(t)=1+\omega t+O(t)$ as $t \rightarrow 0$. Let $B$ and $S(t)$ be as in Theorem 2.4. Furthermore, suppose that $B$ is closed and satisfies

$$
\lim _{t \rightarrow 0^{+}} \frac{T(t) y-y}{t}=-B y \quad \forall y \in Y .
$$

Then for each $x \in Y$, the limit

$$
\lim _{n \rightarrow \infty} T\left(\frac{t}{n}\right)^{n} x=S(t) x
$$

exists uniformly in every bounded interval of $(0, \infty)$. 
Under suitable conditions on the accretiveness and the range, one can prove the closedness of the operator $B$ in Theorems 2.4 and 2.5 .

Remark 2.6. The closedness of the operator $B$ in Theorem 2.4 can be shown as follows. Suppose that $\left\{x_{n}\right\} \subset Y, x_{n} \rightarrow y, B x_{n} \rightarrow z$ as $n \rightarrow \infty$. Then we have to show that $y \in D(B)$ and $B y=z$. In fact, since the inverse $(I+\lambda B)^{-1}$ exists on $Y$ for sufficiently small positive $\lambda$ and is Lipschitz continuous, we have

$$
\begin{aligned}
y & =\lim _{n \rightarrow \infty} x_{n}=\lim _{n \rightarrow \infty}(I+\lambda B)^{-1}(I+\lambda B) x_{n} \\
& =(I+\lambda B)^{-1} \lim _{n \rightarrow \infty}(I+\lambda B) x_{n}=(I+\lambda B)^{-1}(y+\lambda z) .
\end{aligned}
$$

This shows that $y$ is an element of $D(B)$. Furthermore, from this we get

$$
(I+\lambda B) y=(I+\lambda B)(I+\lambda B)^{-1}(y+\lambda z)=y+\lambda z .
$$

Since $\lambda>0$ we have $B y=z$ and the assertion follows.

Now we consider functional differential equations of the form

$$
\frac{d^{+} x}{d t}=f\left(t, x_{t}\right)
$$

where $t \in \mathbb{R}, x \in X$, and $X$ is a Banach space,

$$
\begin{gathered}
f: \mathbb{R} \times C \longrightarrow X, \quad C=C([-r, 0], X), \\
x_{t}(\theta)=x(t+\theta), \quad \theta \in[-r, 0], r>0 .
\end{gathered}
$$

Here and in what follows, we use the notation from [14]. Along with (2.8) we also deal with the Cauchy problem

$$
\frac{d^{+} x}{d t}=f\left(t, x_{t}\right), \quad t \geq s, \quad x_{s}=\phi \in C
$$

is, under some regularity conditions on $f$ (cf. [14]), equivalent to the integral equation

$$
x(t)=\phi(0)+\int_{s}^{t} f\left(\xi, x_{\xi}\right) d \xi, \quad t \geq s, x(\xi)=\phi(\xi-s), \xi \in[s-r, s] .
$$

Definition 2.7. A mapping $f: \mathbb{R} \times C \rightarrow X$ is said to be admissible if the following conditions are satisfied:

(i) $f(t, y)$ is continuous with respect to $(t, y) \in \mathbb{R} \times C$,

(ii) $f(t, y)$ is Lipschitz continuous with respect to $y$ uniformly for $t \in \mathbb{R}$, that is, there exists a positive constant $L$ such that

$$
\|f(t, x)-f(t, y)\|_{X} \leq L\|x-y\|_{C} \quad \forall x, y \in C, t \in \mathbb{R},
$$


(iii) there exists a positive constant $N$ such that

$$
\|f(t, y)\|_{X} \leq N\left(1+\|y\|_{C}\right) \quad \forall(t, y) \in \mathbb{R} \times C .
$$

Theorem 2.8. Let $f$ be admissible. Then the Cauchy problem (2.10) is equivalent to the integral equation (2.11) and it has a unique solution $x_{t}(\phi), t \geq s$ which satisfies the following estimates:

$$
\begin{gathered}
\left\|x_{t}(\phi)\right\| \leq N\left(1+\|\phi\|_{C}\right)\left(1+\frac{e^{L(t-s)}-1}{L}\right), \\
\left\|x_{t}(\phi)-x_{t}(\psi)\right\| \leq e^{L(t-s)}\|\phi-\psi\|_{C} \quad \forall \phi, \psi \in C .
\end{gathered}
$$

Proof. The equivalence of (2.10) and (2.11) as well as the unique existence of solutions is assured by standard arguments (cf. [7, 22]). In order to derive the estimates (2.14) and (2.15), we adapt the corresponding proof from [22]. To this end we first define

$$
\begin{aligned}
& u^{0}(t)=\phi(t-s) \quad \forall s-r \leq t \leq s, \\
& u^{0}(t)=\phi(0) \quad \forall t \geq s,
\end{aligned}
$$

and continue for any $n \in \mathbb{N}$ inductively by setting

$$
\begin{aligned}
& u^{n}(t)=\phi(t-s) \quad \forall s-r \leq t \leq s, \\
& u^{n}(t)=\phi(0)+\int_{s}^{t} f\left(\xi, u_{\xi}^{n-1}\right) d \xi \quad \forall t \geq s .
\end{aligned}
$$

Then the limit

$$
\lim _{n \rightarrow \infty} u^{n}(t)=u(t)
$$

exists uniformly on every compact interval of the form $\left[s, t_{0}\right]$ and $u(t)$ is the unique solution of the Cauchy problem (2.10) (see [22]). From the assumptions we obtain the estimate

$$
\left\|u^{1}(t)-u^{0}(t)\right\| \leq N\left(1+\|\phi\|_{C}\right)(t-s) \quad \forall t \geq s,
$$

and by induction we continue to get for each $n \in \mathbb{N}$,

$$
\left\|u^{n}(t)-u^{n-1}(t)\right\| \leq M L^{n-1} \frac{(t-s)^{n}}{n !} \quad \forall t \geq s,
$$

where $M=N\left(1+\|\phi\|_{C}\right)$. Consequently, we arrive at the estimate

$$
\left\|u_{t}^{n}-u_{t}^{n-1}\right\|_{C} \leq M L^{n-1} \frac{(t-s)^{n}}{n !} \quad \forall t \geq s
$$


which is true for any $n \in \mathbb{N}$. Thus, finally we get

$$
\left\|u_{t}\right\|_{C} \leq M \sum_{\nu=0}^{\infty} L^{\nu-1} \frac{(t-s)^{\nu}}{\nu !}=N\left(1+\|\phi\|_{C}\right)\left(1+\frac{e^{L(t-s)}-1}{L}\right) .
$$

For the proof of (2.15) we refer to [22].

\section{Evolution semigroups associated with nonautonomous functional differential equations}

In this section, we prove a representation theorem for solutions of (2.8), that is the main results of this paper. Throughout we assume that the function $f$ in (2.8) is admissible. Our study is mainly concerned with the so-called evolution semigroup of operators $\left\{T^{h}, h \geq 0\right\}$ associated with (2.8) defined by the formulas

$$
\begin{gathered}
U(t, s) \phi=x_{t}(\phi), \quad \phi \in C, t \geq s, \\
\left(T^{h} v\right)(t, \theta)=[U(t, t-h) v(t-h, \cdot)](\theta), \quad t \in \mathbb{R}, \theta \in[-r, 0], v \in \Delta,
\end{gathered}
$$

where $x_{t}(\phi)$ is determined by (2.10) and $\Delta$ consists of all bounded and uniformly continuous mappings $v: \mathbb{R} \times[-r, 0] \rightarrow X$ with supremum norm. Later on, for every $v \in \Delta$ we will use the abbreviated notation $v(t)=v(t, \cdot)$. Using the existence and the uniqueness of the solutions of (2.10) it is easy to check that $\left\{T^{h}, h \geq 0\right\}$ is indeed a semigroup.

Proposition 3.1. Let f be admissible. Then the family of operators $\left\{T^{h}, h \geq 0\right\}$ defined by (3.2) is a strongly continuous semigroup of operators of type $L$ on $\Delta$, where $L$ is the Lipschitz constant stemming from the admissibility of $f$.

Proof. First of all, we show that for every $h \geq 0$ the operator $T^{h}$ acts on $\Delta$. By definition we have

$$
\left(T^{h} v\right)(t, \theta)=\left\{\begin{array}{l}
v(t-h, 0)+\int_{t-h}^{t+\theta} f(\xi, U(\xi, t-h) v(t-h)) d \xi \quad \text { for } \theta \in[-h, 0] \\
v(t-h, \theta+h) \text { for } \theta \in[-r,-h]
\end{array}\right.
$$

Thus, from (2.14) we get the estimate

$$
\left\|T^{h} v\right\|_{\Delta} \leq\|v\|_{\Delta}+N h\left(1+\|v\|_{\Delta}\right)\left(1+\frac{e^{L h}-1}{L}\right)<\infty
$$

Now we are going to show that $\left(T^{h} v\right)(t, \theta)$ is uniformly continuous with respect to $(t, \theta)$. In fact, we have

$$
\sup _{t, \theta}\left\|\left(T^{h} v\right)(t, \theta)-\left(T^{h} v\right)\left(t^{\prime}, \theta^{\prime}\right)\right\| \leq \max \left\{E_{1}, E_{2}, E_{3}\right\},
$$


where $E_{1}, E_{2}$, and $E_{3}$ are defined by

$$
\begin{gathered}
E_{1}=\sup _{-r \leq \theta, \theta^{\prime} \leq-h}\left\|v(t-h, \theta+h)-v\left(t^{\prime}-h, \theta^{\prime}+h\right)\right\| \\
E_{2}=\sup _{-h \leq \theta, \theta^{\prime} \leq 0} \| v(t-h, 0)-v\left(t^{\prime}-h, 0\right)+\int_{t-h}^{t+\theta} f(\xi, U(\xi, t-h) v(t-h)) d \xi \\
-\int_{t^{\prime}-h}^{t^{\prime}+\theta^{\prime}} f\left(\xi, U\left(\xi, t^{\prime}-h\right) v\left(t^{\prime}-h\right)\right) d \xi \|, \\
E_{3}=\sup _{\theta \leq-h \leq \theta^{\prime}}\left\|v(t-h, \theta+h)-v\left(t^{\prime}-h, 0\right)+\int_{t^{\prime}-h}^{t^{\prime}+\theta^{\prime}} f\left(\xi, U\left(\xi, t^{\prime}-h\right) v\left(t^{\prime}-h\right)\right) d \xi\right\| .
\end{gathered}
$$

Now we let $\left\|(t, \theta)-\left(t^{\prime} \theta^{\prime}\right)\right\|$ tend to 0 . In view of the uniform continuity of $v \in \Delta$ the expressions (3.6) and (3.8) tend to 0 . In order to prove that also (3.7) vanishes, it is sufficient to prove that the limit

$$
\lim _{\left\|(t, \theta)-\left(t^{\prime} \theta^{\prime}\right)\right\| \rightarrow 0} \int_{t-h}^{t+\theta}\left\|U(\xi, t-h) v(t-h)-U\left(\xi, t^{\prime}-h\right) v\left(t^{\prime}-h\right)\right\|_{\Delta} d \xi=0
$$

exists. To this end we suppose, without loss of generality, that $t^{\prime}<t$. Using (2.15) and the admissibility of $f$, we get

$$
\begin{aligned}
\| U(\xi, t-h) v(t-h) & -U\left(\xi, t^{\prime}-h\right) v\left(t^{\prime}-h\right) \|_{C} \\
& \leq h e^{L h}\left\|v(t-h)-U\left(t-h, t^{\prime}-h\right) v\left(t^{\prime}-h\right)\right\|_{C} .
\end{aligned}
$$

Now we state and prove a claim which will be needed in the present proof as well as later on.

Claim 3.2. Under the assumptions and notations of Proposition 3.1, the following holds:

$$
\lim _{h \rightarrow 0^{+}} \sup _{t}\|U(t, t-h) v(t-h)-v(t)\|=0 .
$$

Proof of Claim 3.2. By definition we have

$$
\begin{array}{r}
\sup _{t, \theta}\|[U(t, t-h) v(t-h)](\theta)-v(t, \theta)\| \\
\leq \max \left\{\begin{array}{l}
\sup _{t \in \mathbb{R},-r \leq \theta \leq-h}\|[U(t, t-h) v(t-h)](\theta)-v(t, \theta)\|, \\
\left.\sup _{t \in \mathbb{R},-h \leq \theta \leq 0}\|[U(t, t-h) v(t-h)](\theta)-v(t, \theta)\|\right\} .
\end{array}\right.
\end{array}
$$


The right-hand side of (3.12) equals

$$
\begin{aligned}
& \max \left\{\sup _{t \in \mathbb{R},-r \leq \theta \leq-h}\|v(t-h, \theta+h)-v(t, \theta)\|,\right. \\
& \left.\sup _{t \in \mathbb{R},-h \leq \theta \leq 0}\left\|v(t-h, 0)-v(t, \theta)+\int_{t-h}^{t+\theta} f(\xi, U(\xi, t-h) v(t-h)) d \xi\right\|\right\} .
\end{aligned}
$$

Due to the uniform continuity of $v$, in order to prove (3.11), it is sufficient to show that

$$
\lim _{h \rightarrow 0^{+}} \int_{t-h}^{t+\theta}\|f(\xi, U(\xi, t-h) v(t-h))\|_{C} d \xi=0 .
$$

This relation, however, is clear, because in view of (2.14) we have

$$
\begin{aligned}
\lim _{h \rightarrow 0^{+}} \int_{t-h}^{t+\theta} \| f(\xi, U(\xi, & -h) v(t-h)) \|_{C} d \xi \\
\leq & \lim _{h \rightarrow 0^{+}} N h\left(1+\|v\|_{\Delta}\right)\left(1+\frac{e^{L h}-1}{L}\right)=0 .
\end{aligned}
$$

Thus (3.11) holds and Claim 3.2 is proved.

Now we continue the proof of Proposition 3.1. Obviously, (3.11) implies (3.10) and then (3.9). Thus, the function $T^{h} v(t, \theta)$ is uniformly continuous with respect to $(t, \theta)$ and this means that the operator $T^{h}$ acts on $\Delta$. Next we show that the semigroup $\left\{T^{h}, h \geq 0\right\}$ is strongly continuous. In fact, by definition we have to show that

$$
\lim _{h \rightarrow 0^{+}} \sup _{t, \theta}\left\|\left(T^{h} v\right)(t, \theta)-v(t, \theta)\right\|=0 .
$$

From the definition and the uniform continuity of $v$, it suffices to show that

$$
\lim _{h \rightarrow 0^{+}} \sup _{t,-h \leq \theta \leq 0} \int_{t-h}^{t+\theta}\|f(\xi, U(\xi, t-h) v(t-h))\| d \xi=0 .
$$

This, however, follows from (2.14) and the admissibility of $f$. Thus the proposition is proved.

Definition 3.3. An admissible function $f$ is said to satisfy condition $\mathrm{H}$ if for every $v \in \Delta$ the function taking $t$ into $f(t, v(t, \cdot))$ is uniformly continuous.

Throughout this paper, we use condition $\mathrm{H}$ on $f$ to get the following assertion.

Lemma 3.4. If $f$ satisfies condition $H$, then for every $v \in \Delta$ we get

$$
\lim _{h \rightarrow 0^{+}} \sup _{t}\left\|\frac{1}{h} \int_{t-h}^{t}[f(\xi, U(\xi, t-h) v(t-h))-f(t, v(t))] d \xi\right\|=0 .
$$


Proof. We have

$$
\begin{aligned}
\sup _{t} \| \frac{1}{h} \int_{t-h}^{t}[ & f(\xi, U(\xi, t-h) v(t-h))-f(t, v(t))] d \xi \| \\
\leq & \sup _{t} \frac{1}{h} \int_{t-h}^{t}\|f(\xi, U(\xi, t-h) v(t-h))-f(\xi, v(\xi))\| d \xi \\
& +\sup _{t} \frac{1}{h} \int_{t-h}^{t}\|f(\xi, v(\xi))-f(t, v(t))\| d \xi .
\end{aligned}
$$

Since $f(t, v(t))$ is uniformly continuous with respect to $t$, we get

$$
\lim _{h \rightarrow 0^{+}} \sup _{t} \frac{1}{h} \int_{t-h}^{t}\|f(\xi, v(\xi))-f(t, v(t))\| d \xi=0 .
$$

On the other hand, we have

$$
\begin{aligned}
\sup _{t} \frac{1}{h} \int_{t-h}^{t} \| f(\xi, & U(\xi, t-h) v(t-h))-f(\xi, v(\xi)) \| d \xi \\
\leq & \sup _{t} \frac{1}{h} \int_{t-h}^{t} L\|U(\xi, t-h) v(t-h)-v(\xi)\| d \xi \\
\leq & \sup _{t} \frac{1}{h} \int_{t-h}^{t} L\|U(\xi, t-h) v(t-h)-v(t-h)\| d \xi \\
& +\frac{L}{h} \sup _{t} \int_{t-h}^{t}\|v(t-h)-v(\xi)\| d \xi .
\end{aligned}
$$

Applying (3.11) and taking into account the uniform continuity of $v$ we get (3.18). This completes the proof of the lemma.

Definition 3.5. We define an operator $A$ acting in $\Delta$ as follows:

$$
(A v)(t, \theta)=D v(t, \theta) \stackrel{\text { def }}{=} \lim _{\xi \rightarrow 0,(\theta+\xi \leq 0)} \frac{v(t-\xi, \theta+\xi)-v(t, \theta)}{\xi}
$$

with $D(A)$ consisting of all mappings $v \in \Delta$ such that

(i) $D v(t, \theta)$ exists for all $(t, \theta) \in \mathbb{R} \times[-r, 0]$ and $D v \in \Delta$,

(ii) $(\partial v / \partial t)(t, 0)$ exists and $D v(t, 0)=-(\partial v / \partial t)(t, 0)+f(t, v(t))$,

(iii) $(\partial v / \partial \theta)(t, 0)$ exists and $(\partial v / \partial \theta)(t, 0)=f(t, v(t, \cdot))$ for all $t$.

Remark 3.6. In Definition 3.5 it follows from the properties (i) and (ii) that the function $v$ is differentiable on the line $\mathbb{R} \times\{0\}$ and that then (iii) holds. In order to prove the differentiability of $v$, we put

$$
g(s)=v(t+\xi+\zeta-s, s)-v(t+\xi+\zeta, 0)-h D v(t, 0) \quad \text { for } s \leq 0 .
$$

A simple computation provides the identity $g(0)=0$ as well as

$$
g^{\prime}(s)=D v(t+\xi+\zeta-s, s)-D v(t, 0) .
$$


Hence, we obtain the estimate

$$
\|g(s)\| \leq|s| \sup _{x \in[s, 0]}\|D v(t+\xi+\zeta-x, x)-D v(t, 0)\| .
$$

Putting $s=\zeta$, we get

$$
\begin{aligned}
\| v(t+\xi, \zeta) & -v(t+\xi+\zeta, 0)-\zeta D v(t, 0) \| \\
& \leq|\zeta| \sup _{x \in[\zeta, 0]}\|D v(t+\xi+\zeta-x, x)-D v(t, 0)\| .
\end{aligned}
$$

Similarly, we obtain

$$
\begin{aligned}
& \left\|v(t+\xi+\zeta, 0)-v(t, 0)-(\xi+\zeta) \frac{\partial v}{\partial t}(t, 0)\right\| \\
& \quad \leq|\xi+\zeta| \sup _{x \in[0, \xi+\zeta]}\left\|\frac{\partial v}{\partial t}(t+\xi+\zeta-x, 0)-\frac{\partial v}{\partial t}(t, 0)\right\| .
\end{aligned}
$$

Finally, we get the estimate

$$
\begin{aligned}
& \| v(t+\xi, \zeta)-v(t, 0)-\zeta\left(D v(t, 0)+\frac{\partial v}{\partial t}(t, 0)\right)-\xi \frac{\partial v}{\partial t}(t, 0) \| \\
&=\left\|v(t+\xi, \zeta)-v(t, 0)-\zeta D v(t, 0)-(\xi+\zeta) \frac{\partial v}{\partial t}(t, 0)\right\| \\
& \leq\|v(t+\xi, \zeta)-v(t+\xi+\zeta, 0)\|+\|v(t+\xi+\zeta, 0)-v(t, 0)\| \\
& \leq(|\xi|+|\zeta|) \max \left\{\sup _{x \in[\zeta, 0]}\|D v(t+\xi+\zeta-x, x)-D v(t, 0)\|,\right. \\
&\left.\sup _{x \in[0, \xi+\zeta]}\left\|\frac{\partial v}{\partial t}(t+\xi+\zeta-x, 0)-\frac{\partial v}{\partial t}(t, 0)\right\|\right\} .
\end{aligned}
$$

In view of this relation and the uniform continuity of $D v(t, \theta)$ with respect to $(t, \theta)$, we observe that $v$ is differentiable on $\mathbb{R} \times\{0\}$. Consequently, we get

$$
\begin{aligned}
D v(t, 0) & =-\frac{\partial v}{\partial t}(t, 0)+\frac{\partial v}{\partial \theta}(t, 0) \\
& =-\frac{\partial v}{\partial t}(t, 0)+f(t, v(t)), \quad \frac{\partial v}{\partial \theta}(t, 0)=f(t, v(t)) .
\end{aligned}
$$

Proposition 3.7. Let $f$ satisfy condition $H$. Then $A$ is the infinitesimal generator of the strongly continuous semigroup $\left\{T^{h}, h \geq 0\right\}$.

Proof. Suppose that $v \in D(B)$, where $B$ is the infinitesimal generator of $\left\{T^{h}, h \geq 0\right\}$. We have to show that $v \in D(A)$ and $B v=A v$. In fact, by definition $v$ belongs to $D(B)$ if and only if

$$
\lim _{h \rightarrow 0^{+}} \frac{T^{h} v-v}{h}=B v \in \Delta .
$$


From the definition of $T^{h}$, it is clear that for every $\theta<0$ we get $B v(t, \theta)=D v(t, \theta)$. Since $B v$ is an element of $\Delta$ it is not difficult to show that $D v(t, \theta)$ exists for all $(t, \theta) \in \mathbb{R} \times[-r, 0]$ using the following elementary claim following from the mean value theorem.

Claim 3.8. Let $f:[a, b] \rightarrow X$ be a continuous function which is continuously differentiable on $[a, b)$ such that

$$
\lim _{x \rightarrow b^{-}} f^{\prime}(x)=c .
$$

Then $f$ is differentiable from the left at $b$ with derivative $f^{\prime}(b)=c$.

Continuing the proof of Proposition 3.7 we put $\theta=0$. Then by definition, we get

$$
B(t, 0)=\lim _{h \rightarrow 0^{+}}\left[\frac{v(t-h, 0)-v(t, 0)}{h}+\frac{1}{h} \int_{t-h}^{t} f(\xi, U(\xi, t-h) v(t-h)) d \xi\right] .
$$

By virtue of Lemma 3.4, the derivative $(\partial v / \partial t)(t, 0)$ exists and is uniformly continuous. Furthermore, we get

$$
B v(t, 0)=-\frac{\partial v}{\partial t}(t, 0)+f(t, v(t)) .
$$

Thus $v$ is an element of $D(A)$ and $B v=A v$. Conversely, supposing $v \in D(A)$ we now show that $v \in D(B)$ and $A v=B v$. In fact, by definition we have to show that

$$
\begin{aligned}
& \sup _{t, \theta}\left\|\left[\frac{T^{h} v-v}{h}-A v\right](t, \theta)\right\| \\
&=\max \left\{\sup _{t,-r \leq \theta \leq-h}\left\|\frac{v(t-h, \theta+h)-v(t, \theta)}{h}-A v(t, \theta)\right\|,\right. \\
&\left.\quad \sup _{-h \leq \theta \leq 0}\left\|\frac{v(t-h, 0)-v(t, \theta)+\int_{t-h}^{t+\theta} f(\xi, U(\xi, t-h) v(t-h)) d \xi}{h}-A v(t, \theta)\right\|\right\} .
\end{aligned}
$$

Using the uniform continuity of $D v(t, \theta)$, it is not difficult to see that

$$
\begin{aligned}
\lim _{h \rightarrow 0^{+}} \sup _{t,-r \leq \theta \leq-h} \| & \left\|\frac{v(t-h, \theta+h)-v(t, \theta)}{h}-A v(t, \theta)\right\| \\
& \leq \lim _{h \rightarrow 0^{+}} \sup _{0 \leq \xi \leq h}\|D v(t-h, \theta+h)-D v(t, \theta)\|=0 .
\end{aligned}
$$

Since $v \in D(A)$ we have

$$
D v(t, 0)=-\frac{\partial v}{\partial t}(t, 0)+f(t, v(t)) .
$$

Consequently, since $f$ satisfies condition $\mathrm{H}$, the function $(\partial v / \partial t)(t, 0)$ is bounded and uniformly continuous on $\mathbb{R} \times\{0\}$. Thus, it is easy to show that

$$
\lim _{h \rightarrow 0^{+}} \sup _{t}\left\|\frac{v(t-h, 0)-v(t, 0)}{h}+\frac{\partial v}{\partial t}(t, 0)\right\|=0 .
$$


We have

$$
\begin{aligned}
\sup _{t,-h \leq \theta \leq 0} & \left\|\frac{v(t-h, 0)-v(t, \theta)+\int_{t-h}^{t+\theta} f(\xi, U(\xi, t-h) v(t-h))}{h}-A v(t, \theta)\right\| \\
\leq & \sup _{t,-h \leq \theta \leq 0}\left\|\frac{v(t-h, 0)-v(t, \theta)}{h}+\int_{t-h}^{t+\theta} \frac{f(\xi, U(\xi, t-h) v(t-h))}{h}-A v(t, 0)\right\| \\
& +\sup _{-h \leq \theta \leq 0}\|A(t, 0)-A(t, \theta)\| \\
\leq & \sup _{t}\left\|\frac{v(t-h, 0)-v(t, 0)}{h}+\frac{\partial v}{\partial t}(t, 0)\right\| \\
& +\sup _{t,-h \leq \theta \leq 0} \frac{1}{h}\left\|v(t, 0)-v(t, \theta)+\left(\int_{t-h}^{t+\theta}+\int_{t-h}^{t}\right) f(\xi, U(\xi, t-h) v(t-h)) d \xi\right\| \\
& +\left\|f(t, v(t))-\int_{t-h}^{t} f(\xi, U(\xi, t-h) v(t-h)) d \xi\right\| .
\end{aligned}
$$

According to Lemma 3.4, we then get

$$
\lim _{h \rightarrow 0^{+}} \sup _{t}\left\|f(t, v(t))-\frac{1}{h} \int_{t-h}^{t} f(\xi, U(\xi, t-h) v(t-h)) d \xi\right\|=0 .
$$

From the uniform continuity of the functions $D v(t, \theta)$ and $(\partial v / \partial t)(t, 0)$ and in view of the relations (3.18) and (3.28), we have

$$
\lim _{\theta \rightarrow 0^{-}} \sup _{t}\left\|\frac{v(t, \theta)-v(t, 0)}{\theta}-f(t, v(t))\right\|=0 .
$$

Thus, we get

$$
\begin{aligned}
\frac{1}{h} \| v(t, 0) & -v(t, \theta)+\int_{t+\theta}^{t} f(\xi, U(\xi, t-h) v(t-h)) d \xi \| \\
\leq & -\frac{\theta}{h}\left\|\frac{v(t, \theta)-v(t, 0)}{\theta}-\frac{1}{\theta} \int_{t+\theta}^{t} f(\xi, U(\xi, t+\theta) v(t+\theta)) d \xi\right\| \\
& +\frac{1}{\theta} \int_{t+\theta}^{t}\|f(\xi, U(\xi, t+\theta) v(t+\theta))-f(\xi, U(\xi, t-h) v(t-h))\| d \xi
\end{aligned}
$$

It may be concluded from (2.14), (2.15), and (3.11) that

$$
\lim _{\theta \rightarrow 0^{-}} \sup _{t} \frac{1}{\theta} \int_{t+\theta}^{t}\|f(\xi, U(\xi, t+\theta) v(t+\theta))-f(\xi, U(\xi, t-h) v(t-h))\| d \xi=0 .
$$

On the other hand, in view of (3.40) and Lemma 3.4 we have

$$
\lim _{\theta \rightarrow 0^{-}} \sup _{t}\left\|\frac{v(t, \theta)-v(t, 0)}{\theta}-\frac{1}{\theta} \int_{t+\theta}^{t} f(\xi, U(\xi, t+\theta) v(t+\theta)) d \xi\right\|=0 .
$$


Now combining (3.37), (3.42), and (3.43) and using the uniform continuity of $D v(t, \theta)$, we observe that

$$
\lim _{h \rightarrow 0^{+}}\left\|\frac{T^{h} v-v}{h}-A v\right\|=0 .
$$

This finally means that $v \in D(B)$ and $B v=A v$. Therefore, the proof of the proposition is complete.

Now we continue to study the operator $A$ by proving the following assertion.

Proposition 3.9. Let A be defined by Definition 3.5. Furthermore, let $f$ satisfy condition $H$. Then for $0<\lambda<1 / L$ the range of the operator $I-\lambda A$ equals $\Delta$, the inverse $(I-\lambda A)^{-1}$ exists and is Lipschitz continuous with Lipschitz constant $(1-\lambda L)^{-1}$. In particular, the operator $(L I-A)$ is accretive.

Proof. Assuming that $\phi \in \Delta$, we are going to show that there exists a unique $\psi \in D(A)$ such that

$$
\psi-\lambda A \psi=\phi
$$

By definition, it may be seen that $\psi$ belongs to $D(A)$ and that $\psi$ solves (3.45) if and only if

$$
\begin{gathered}
\psi(t, \theta)-\lambda D \psi(t, \theta)=\phi(t, \theta) \quad \forall(t, \theta) \in \mathbb{R} \times[-r, 0), \\
\psi(t, 0)+\lambda \frac{\partial \psi}{\partial t}(t, 0)-\lambda f(t, \psi(t, \cdot))=\phi(t, 0) \quad \forall t \in \mathbb{R} .
\end{gathered}
$$

We first solve (3.46) by the method of characteristics and then show that $\psi$ belongs to $D(A)$. Setting

$$
w(\zeta) \stackrel{\text { def }}{=} \psi(t+\theta-\zeta, \zeta)
$$

we see that $w(\theta)=\psi(t, \theta)$ and $w(0)=\psi(t+\theta, 0)$ as well as

$$
w^{\prime}(\zeta)=\frac{d w(\zeta)}{d \zeta}=D \psi(t+\theta-\zeta, \zeta)
$$

Thus from the equation

$$
\psi(t+\theta-\zeta, \zeta)-\lambda D \psi(t+\theta-\zeta, \zeta)=\phi(t+\theta-\zeta, \zeta),
$$

we get

$$
w(\zeta)-\lambda w^{\prime}(\zeta)=\phi(t+\theta-\zeta, \zeta)
$$

Now solving (3.50), we obtain

$$
\begin{aligned}
\psi(t, \theta) & =w(\theta)=w(0) e^{\theta / \lambda}-\frac{1}{\lambda} \int_{0}^{\theta} e^{(\theta-m) / \lambda} \phi(t+\theta-m, m) d m \\
& =\psi(t+\theta, 0) e^{\theta / \lambda}-\frac{1}{\lambda} \int_{0}^{\theta} e^{(\theta-m) / \lambda} \phi(t+\theta-m, m) d m
\end{aligned}
$$


On the other hand, since $\psi(t, 0)$ is the bounded solution of the equation

$$
\frac{d x}{d t}=-\frac{1}{\lambda} x+\left(f(t, \psi(t, \cdot))+\frac{1}{\lambda} \phi(t, 0)\right), \quad x \in X, t \in \mathbb{R},
$$

$\psi(t, 0)$ has the form

$$
\int_{-\infty}^{t} e^{-(1 / \lambda)(t-\xi)}\left[f(\xi, \psi(\xi, \cdot))+\frac{1}{\lambda} \phi(\xi, 0)\right] d \xi
$$

Finally, for $\psi$ we get the integral equation

$$
\begin{aligned}
\psi(t, \theta)= & \int_{-\infty}^{t+\theta} e^{-(1 / \lambda)(t-\xi)}\left[f(\xi, \psi(\xi, \cdot))+\frac{1}{\lambda} \phi(\xi, 0)\right] d \xi \\
& +-\frac{1}{\lambda} \int_{0}^{\theta} e^{(\theta-m) / \lambda} \phi(t+\theta-m, m) d m .
\end{aligned}
$$

Now, we solve (3.54) by considering an operator $K$ acting on $\Delta$ where $K \psi(t, \theta)$ is defined as the right-hand side of (3.54). It is clear that $K \psi \in \Delta$. Furthermore, we have

$$
\begin{aligned}
\|K u-K v\| & \leq \sup _{t, \theta} \int_{-\infty}^{t+\theta} e^{-(1 / \lambda)(t-\xi)}\|f(\xi, u(\xi))-f(\xi, v(\xi))\| d \xi \\
& \leq \sup _{t, \theta} \int_{-\infty}^{t+\theta} e^{-(1 / \lambda)(t-\xi)} L\|u(\xi)-v(\xi)\| d \xi \\
& \leq(\lambda L) e^{\theta / \lambda}\|u-v\|_{\Delta}=\lambda L\|u-v\|_{\Delta} .
\end{aligned}
$$

Hence, $K$ is a strict contraction which therefore has a unique fixed point. For simplicity we denote it by $\psi$ as well. Now, it is not difficult to show that $\psi$ as a unique solution of (3.54) is also a unique solution of (3.46). This shows that for $0<\lambda<L$ we get $R(I-\lambda A)=\Delta$ and that $(I-\lambda A)^{-1}$ exists (as a single-valued operator on $\left.\Delta\right)$. Now we are going to show that $(I-\lambda A)^{-1}$ is Lipschitz continuous with constant $(1-\lambda L)^{-1}$. To this end let $\psi_{1}, \psi_{2}$ be the solutions of (3.54) corresponding to $\phi_{1}, \phi_{2}$, respectively. Therefore, we have

$$
\begin{aligned}
\left\|\psi_{1}-\psi_{2}\right\|_{\Delta} \leq \sup _{t, \theta}\left\{\int_{\infty}^{t+\theta} e^{-(1 / \lambda)(t-\xi)} L\left\|\psi-\psi_{2}\right\|_{\Delta} d \xi\right. \\
+\frac{1}{\lambda} \int_{-\infty}^{t+\theta} e^{-(1 / \lambda)(t-\xi)}\left\|\phi_{1}-\phi_{2}\right\|_{\Delta} d \xi \\
\left.\quad+\int_{0}^{\theta} e^{(1 / \lambda)(\theta-\xi)} \frac{1}{\lambda}\left\|\phi_{1}-\phi_{1}\right\|_{\Delta} d \xi\right\} \\
\leq \lambda L\left\|\psi_{1}-\psi_{2}\right\|_{\Delta}+\left\|\phi_{1}-\phi_{2}\right\|_{\Delta} .
\end{aligned}
$$

Thus, we get

$$
\begin{aligned}
\left\|\psi_{1}-\psi_{2}\right\|_{\Delta} & =\left\|(I-\lambda A)^{-1} \phi_{1}-(I-\lambda A)^{-1} \phi_{2}\right\| \\
& \leq \frac{1}{1-\lambda L}\left\|\phi_{1}-\phi_{2}\right\|_{\Delta} \quad \forall \phi_{1}, \phi_{2} \in \Delta .
\end{aligned}
$$

This completes the proof of the proposition. 
Proposition 3.10. If $f$ satisfies condition $H$, then $A$ is densely defined in $\Delta$.

Proof. It is sufficient to show that

$$
\lim _{\lambda \rightarrow 0^{+}}(I-\lambda A)^{-1} \phi=\phi
$$

for every given $\phi \in \Delta$. It may be noted that (3.58) holds for the linear case, in particular, if $f(t, y)=0$ for all $t$ and $y$. Therefore, we have

$$
\begin{aligned}
& \lim _{\lambda \rightarrow 0^{+}} \sup _{t, \theta}\left\|\phi_{\lambda}(t, \theta)-\phi(t, \theta)\right\| \\
&=\lim _{\lambda \rightarrow 0^{+}} \sup _{t, \theta} \| \int_{-\infty}^{t+\theta} e^{-(1 / \lambda)(t-\xi)} \frac{1}{\lambda} \phi(\xi, 0) d \xi \\
& \quad-\int_{0}^{\theta} e^{(1 / \lambda)(\theta-m)} \frac{1}{\lambda} \phi(t+\theta-m, m) d m-\phi(t, \theta) \|=0 .
\end{aligned}
$$

Thus it is sufficient to show that

$$
\lim _{\lambda \rightarrow 0^{+}}\left\|\psi_{\lambda}-\phi_{\lambda}\right\|=0,
$$

where $\psi_{\lambda}$ denotes the solution of the integral equation (3.54). Since $\psi_{\lambda}$ is the unique fixed point of the operator $K$, we have

$$
\begin{aligned}
\left\|\psi_{\lambda}-\phi_{\lambda}\right\|_{\Delta} & \leq\left\|K \psi_{\lambda}-K \phi_{\lambda}\right\|_{\Delta}+\left\|K \phi_{\lambda}-\phi_{\lambda}\right\|_{\Delta} \\
& \leq \lambda L\left\|\psi_{\lambda}-\phi_{\lambda}\right\|_{\Delta}+\left\|K \phi_{\lambda}-\phi_{\lambda}\right\|_{\Delta} .
\end{aligned}
$$

Thus,

$$
\left\|\psi_{\lambda}-\phi_{\lambda}\right\|_{\Delta} \leq \frac{1}{1-\lambda L}\left\|K \phi_{\lambda}-\phi_{\lambda}\right\|_{\Delta} .
$$

On the other hand, we have

$$
\begin{aligned}
\left\|K \phi_{\lambda}-\phi_{\lambda}\right\|_{\Delta} & \leq \sup _{t, \theta} \int_{-\infty}^{t+\theta} e^{-(1 / \lambda)(t-\xi)}\|f(\xi, \phi(\xi))\| d \xi \\
& \leq \int_{-\infty}^{t} e^{-(1 / \lambda)(t-\xi)} N\left(1+\|\phi\|_{\Delta}\right) d \xi=\lambda N\left(1+\|\phi\|_{\Delta}\right) .
\end{aligned}
$$

This shows that (3.58) holds. The proof of the proposition is therefore complete.

Now we are in a position to apply Theorems 2.4 and 2.5 in order to get the main result of this section.

THEOREM 3.11 (representation theorem). If $f$ satisfies condition $H$, then the operator A generates the semigroup $\left\{T^{h}, h \geq 0\right\}$ defined by (3.2) in the Crandall-Liggett sense, that is,

$$
\lim _{n \rightarrow \infty}\left(I-\frac{h}{n} A\right)^{-n} v=T^{h} v \quad \forall v \in \Delta
$$


Furthermore, $T^{h}$ represents the solution $x_{t}(\phi)$ of (2.10) in the sense that

$$
x_{t}(\phi)=\left(T^{t-s} \phi\right)(t) \quad \forall \phi \in \Delta .
$$

Proof. The theorem is a direct consequence of Propositions 3.1, 3.7, and 3.9 and Theorems 2.4 and 2.5 .

\section{Bounded and almost periodic solutions and evolution semigroups}

In this section, we apply Theorem 3.11 to study the asymptotic behavior of solutions of the functional differential equation (2.8).

THEOREM 4.1. Let $f$ satisfy condition $H$ and let $\omega$ be a negative number such that $\omega I-A$ is accretive. Then (2.8) has a unique solution which is defined on $\mathbb{R}$ and bounded as well as exponentially stable.

Proof. According to Theorem 3.11, the accretiveness of $\omega I-A$ implies (3.64). Therefore there exists a unique fixed point $\psi$ for any $T^{h}, h \geq 0$. Obviously, $\psi$ belongs to $D(A)$ and $A \psi=0$. Consequently, we get $\psi(t, \theta)=\psi(t+\theta, 0)$. Setting $x(t)=\psi(t, 0)$, we obtain $x_{t}(\theta)=\psi(t+\theta, 0)=\psi(t, \theta)$. Since $A \psi=0$ it follows that

$$
\frac{d^{+} x}{d t}=\frac{\partial \psi}{\partial t}(t, 0)=f(t, \psi(t, \cdot))=f(t, \psi(t+\cdot, 0))=f\left(t, x_{t}\right) .
$$

Now, using (3.64) and (3.65) we see that every solution $y_{t}(\phi)$ of the Cauchy problem (2.10) satisfies

$$
\begin{aligned}
\left\|x_{t}-y_{t}\right\|_{C} & =\left\|U(t, s) x_{s}-U(t, s) \phi\right\|_{C} \\
& =\left\|\left(T^{t-s} \psi\right)(t)-\left(T^{t-s} \phi^{*}\right)(t)\right\|_{C} \\
& \leq e^{\omega(t-s)}\|\phi-\psi\|_{\Delta},
\end{aligned}
$$

where $\phi^{*}(t, \theta) \stackrel{\text { def }}{=} \phi(\theta)$ for all $(t, \theta)$. This completes the proof of the theorem.

Remark 4.2. As noted in the introduction, the condition of Theorem 4.1 on the accretiveness of the operator $\omega I-A$ in the case of equations without delay turns out to be that of the differential operator $\omega I-\{-d / d t+f(t, \cdot)\}$ in the corresponding function space. A sufficient condition for this is the accretiveness of the operator $\{\omega I-f(t, \cdot)\}$ in the phase space $X$. In fact, this can be easily proved by using a fundamental result on continuous perturbation of linear accretive operator (see [23]). Here, it may be noted that the operator $-d / d t$ in the function space $C_{u}(\mathbb{R}, X)$ of uniformly continuous and bounded $X$-valued functions is accretive. Hence, Theorem 4.1 is a FDE-analog of Medvedev's result (see [10, 12, 16, 20, 21]).

A consequence of the representation theorem and Theorem 4.1 is the following which concerns the almost periodicity. To this end we recall the following notion. 
Definition 4.3. A function $u: \mathbb{R} \rightarrow Y$ is said to be almost periodic if for every given sequence $\left\{\tau_{n}\right\}_{n \in \mathbb{N}}$, there exists a subsequence $\left\{\tau_{n_{k}}\right\}_{k \in \mathbb{N}}$ such that the sequence of functions $\left\{u\left(\cdot+\tau_{n_{k}}\right)\right\}_{k \in \mathbb{N}}$ is uniformly convergent on $\mathbb{R}$ as $k \rightarrow \infty$.

COROLlary 4.4. Let all assumptions of Theorem 4.1 be satisfied. Furthermore, for every fixed $y$, let $f(t, y)$ be almost periodic with respect to $t$. Then (2.8) has a unique exponentially stable solution which is almost periodic.

Proof. We define the following closed subspace:

$$
\Delta_{a p}=\{v \in \Delta: v(\cdot) \text { is almost periodic }\} .
$$

We will use the representation theorem to show that $\left\{T^{h}, h \geq 0\right\}$ leaves $\Delta_{a p}$ invariant, that is, for every $\phi \in \Delta_{a p}$ we have $T^{h} \phi \in \Delta_{a p}$. In fact, we first show that

$$
(I-\lambda A)^{-1} \phi \in \Delta_{a p} \text { for } 0<\lambda<\frac{1}{L} .
$$

We return to the integral equation (3.54) which determines $\psi$ as the solution of the integral equation (3.54) with $\psi=(I-\lambda A)^{-1} \phi$. Now we show that $\psi$ is almost periodic. To this end, it suffices to prove that the integral operator defined by the right-hand side of (3.54) (that is, the operator $K$ ) leaves $\Delta_{a p}$ invariant. But this can be easily seen by considering the inhomogeneous equation

$$
\frac{d y}{d \tau}=-\frac{1}{\lambda} y+g(\tau),
$$

where

$$
g(\tau)=f(\tau, \psi(\tau, \cdot))+\frac{1}{\lambda} \phi(\tau, 0) .
$$

Note that $g(\tau)$ is almost periodic with respect to $\tau$. In fact, this is equivalent to the almost periodicity of $f(\tau, u(\tau, \cdot))$ for every fixed $u \in \Delta_{a p}$. In turn, this can be proved in the same way as in [4, Chapter VII, Lemma 4.1]. According to the representation theorem,

$$
T^{h} \phi=\lim _{n \rightarrow \infty}\left(I-\frac{h}{n} A\right)^{-n} \phi \in \Delta_{a p} .
$$

Now we are in a position to apply Theorem 4.1 to see that the unique fixed point of the semigroup $\left\{T^{h}, h \geq 0\right\}$ should be in $\Delta_{a p}$. This completes the proof.

In conclusion we remark that Theorem 4.1 and Corollary 4.4 are closely related to recent results by S. Kato et al., see for example, [11, 12], and also Seifert [20, 21], Kartsatos [10] in which a result by Medvedev [16], similar to Theorem 4.1 but for equations without delay, plays the key role to prove the existence and uniqueness of the bounded solution to the underlying equation. Our constructions can be easily extended to the case in which the semigroup $\left\{T^{h}, h \geq 0\right\}$ leaves invariant some subspace of $\Delta_{a p}$. This subspace can be determined as in the proof of Corollary 4.4. A simple model of this is the subspace of periodic functions. 


\section{Acknowledgement}

The second author was supported by the Alexander von Humboldt Foundation. The assistance of the Foundation is gratefully acknowledged.

\section{References}

[1] B. Aulbach and N. V. Minh, Nonlinear semigroups and the existence and stability of solutions of semilinear nonautonomous evolution equations, Abstr. Appl. Anal. 1 (1996), no. 4, 351-380. MR 98k:47135. Zbl 934.34051.

[2] H. Brézis and A. Pazy, Convergence and approximation of semigroups of nonlinear operators in Banach spaces, J. Functional Analysis 9 (1972), 63-74. MR 45\#2529. Zbl 231.47036.

[3] M. G. Crandall and T. M. Liggett, Generation of semi-groups of nonlinear transformations on general Banach spaces, Amer. J. Math. 93 (1971), 265-298. MR 44\#4563. Zbl 226.47038.

[4] J. L. Daleckii and M. G. Krein, Stability of Solutions of Differential Equations in Banach Space, vol. 43, American Mathematical Society, Rhode Island, 1974, translated from the Russian by S. Smith. Translations of Mathematical Monographs. MR 50\#5126. Zbl 286.34094.

[5] J. Dyson and R. V. Bressan, Functional differential equations and non-linear evolution operators, Proc. Roy. Soc. Edinburgh Sect. A 75 (1975/76), no. 3, 223-234. MR 56\#784. Zbl 361.34055.

[6] A. M. Fink, Almost Periodic Differential Equations, vol. 377, Springer-Verlag, Berlin, 1974, Lecture Notes in Mathematics. MR 57\#792. Zbl 325.34039.

[7] J. K. Hale and S. M. Verduyn Lunel, Introduction to Functional-Differential Equations, Applied Mathematical Sciences, vol. 99, Springer-Verlag, New York, 1993. MR 94m:34169. Zbl 787.34002.

[8] Y. Hino, S. Murakami, and T. Naito, Functional-Differential Equations with Infinite Delay, vol. 1473, Springer-Verlag, Berlin, 1991, Lecture Notes in Mathematics. MR 92g:34088. Zbl 732.34051.

[9] F. Kappel, Semigroups and delay equations, Semigroups, Theory and Applications, Pitman Res. Notes Math. Ser., vol. 152, Longman Sci. Tech., Harlow, 1986, (Trieste, 1984), pp. 136-176. MR 88d:34093. Zbl 622.47041.

[10] A. G. Kartsatos, Almost periodic solutions to nonlinear systems, Boll. Un. Mat. Ital. (4) 9 (1974), 10-15. MR 50\#7677. Zbl 295.34028.

[11] S. Kato, Almost periodic solutions of functional-differential equations with infinite delays in a Banach space, Hokkaido Math. J. 23 (1994), no. 3, 465-474. MR 95j:34116. Zbl 819.34048.

[12] S. Kato and M. Imai, On the existence of periodic solutions and almost periodic solutions for nonlinear systems, Nonlinear Anal. 24 (1995), no. 8, 1183-1192. MR 96c:34067. Zbl 834.34045.

[13] Y. Latushkin and S. Montgomery-Smith, Evolutionary semigroups and Lyapunov theorems in Banach spaces, J. Funct. Anal. 127 (1995), no. 1, 173-197. MR 96k:47072. Zbl 878.47024.

[14] Y. Latushkin, S. Montgomery-Smith, and T. Randolph, Evolutionary semigroups and dichotomy of linear skew-product flows on locally compact spaces with Banach fibers, J. Differential Equations 125 (1996), no. 1, 73-116. MR 97a:47056. Zbl 881.47020.

[15] Y. Latushkin and T. Randolph, Dichotomy of differential equations on Banach spaces and an algebra of weighted translation operators, Integral Equations Operator Theory 23 (1995), no. 4, 472-500. MR 96j:47034. Zbl 839.47026. 
[16] N. V. Medvedev, Certain criteria for the existence of bounded solutions of a system of differential equations, Differencial'nye Uravnenija 4 (1968), 1258-1264 (Russian). MR 37\#6536.

[17] N. V. Minh, Semigroups and stability of nonautonomous differential equations in Banach spaces, Trans. Amer. Math. Soc. 345 (1994), no. 1, 223-241. MR 95a:34091. Zbl 820.34039.

[18] T. Naito and N. V. Minh, Evolution semigroups and spectral criteria for almost periodic solutions of periodic evolution equations, J. Differential Equations 152 (1999), no. 2, 358-376. MR 99m:34131. Zbl 924.34038.

[19] A. G. Nickel, On evolution semigroups and wellposedness of nonautonomous Cauchy problems, Ph.D. thesis, Tuebingen, 1996. Zbl 880.47024.

[20] G. Seifert, Almost periodic solutions for delay-differential equations with infinite delays, J. Differential Equations 41 (1981), no. 3, 416-425. MR 83a:34109. Zbl 461.34053.

[21] Almost periodic solutions for a certain class of almost periodic systems, Proc. Amer. Math. Soc. 84 (1982), no. 1, 47-51. MR 83a:34060. Zbl 486.34026.

[22] C. C. Travis and G. F. Webb, Existence and stability for partial functional differential equations, Trans. Amer. Math. Soc. 200 (1974), 395-418. MR 52\#3690. Zbl 299.35085.

[23] G. F. Webb, Continuous nonlinear perturbations of linear accretive operators in Banach spaces, J. Functional Analysis 10 (1972), 191-203. MR 50\#14407. Zbl 245.47052.

[24] Autonomous nonlinear functional differential equations and nonlinear semigroups, J. Math. Anal. Appl. 46 (1974), 1-12. MR 50\#722. Zbl 277.34070.

[25] T. Yoshizawa, Stability Theory and the Existence of Periodic Solutions and Almost Periodic Solutions, vol. 14, Springer-Verlag, New York, 1975, Applied Mathematical Sciences. MR 57\#6673. Zbl 304.34051.

B. Aulbach: Department of Mathematics, University of Augsburg, D-86135 Augsburg, Germany

E-mail address: aulbach@math.uni-augsburg.de

N. Van Minh: Department of Mathematics, The University of ElectroCommunications, Chofugaoka 1-5-1, Chofu, TOKyo 182-8585, JaPan

E-mail address: minh@matha.e-one.uec.ac.jp 


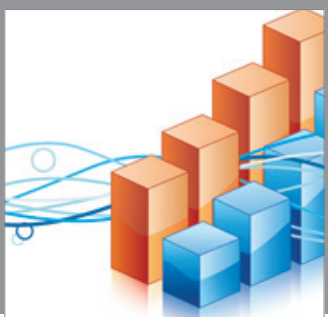

Advances in

Operations Research

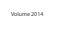

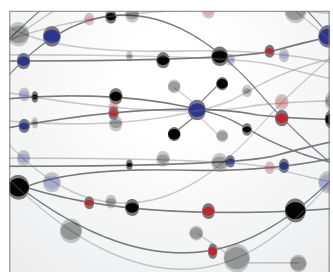

\section{The Scientific} World Journal
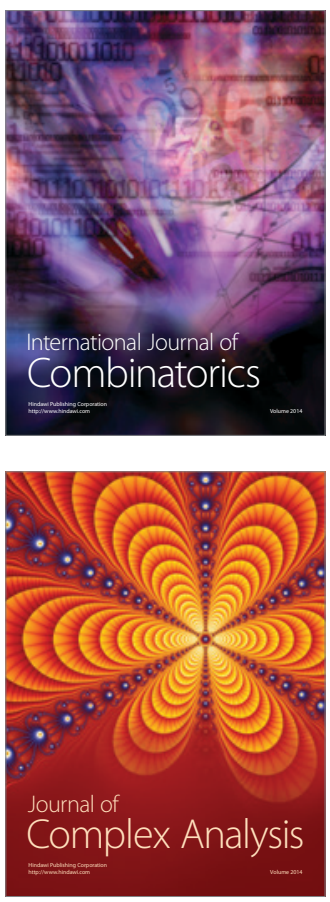

International Journal of

Mathematics and

Mathematical

Sciences
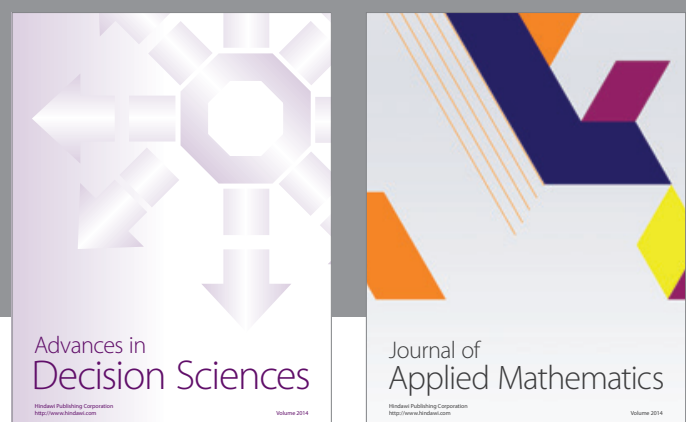

Journal of

Applied Mathematics
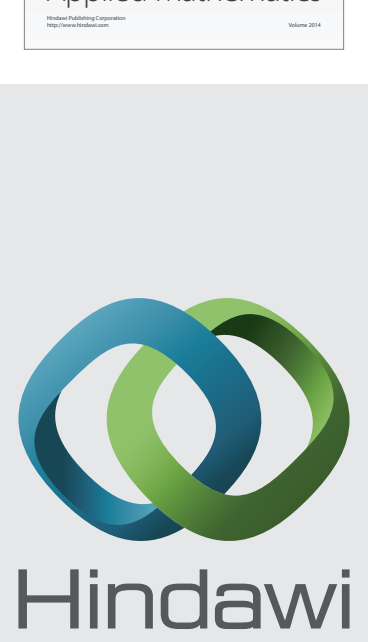

Submit your manuscripts at http://www.hindawi.com
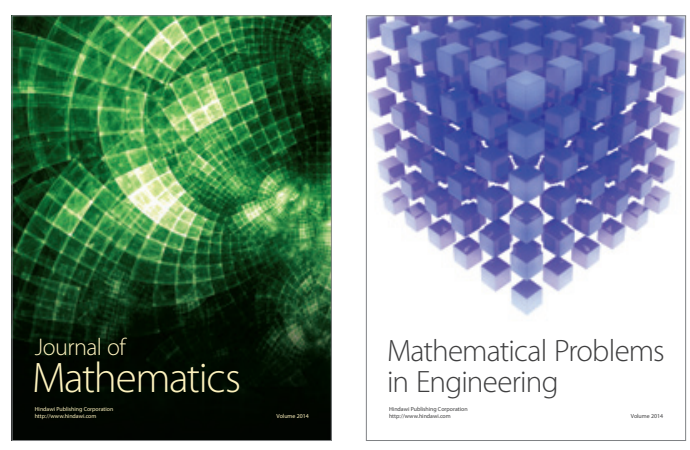

Mathematical Problems in Engineering
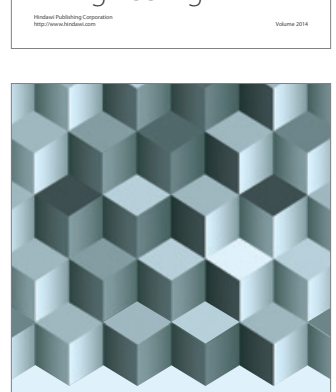

Journal of

Function Spaces
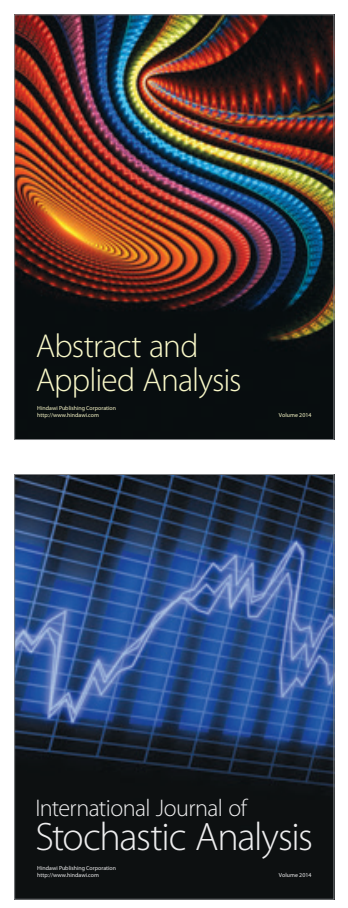

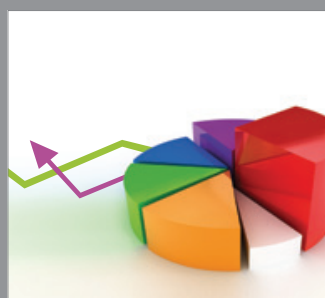

ournal of

Probability and Statistics

Promensencen
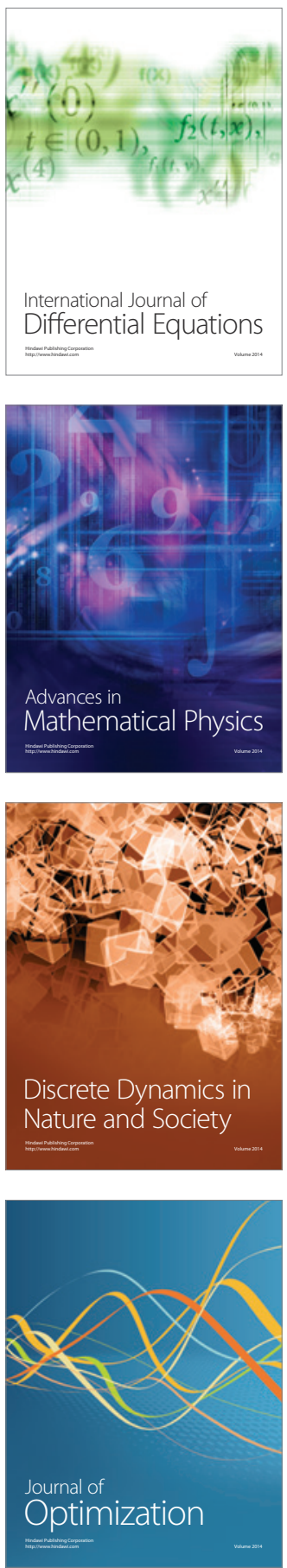